\title{
Nuestra herencia de maíz: Semillas nativas vs. semillas transgénicas, una aproximación desde la cultura y los derechos humanos
}

Our corn heritage: native and transgenic seeds, an approach from culture and human rights

Autores: Yesica Ruiz Cristancho, Ricardo Alonso Tejedor Estupiñán

DOI: https://doi.org/10.19053/16923936.v18.n35.2020.10183

Para citar este artículo:

Ruiz-Cristancho, Y. y Tejedor-Estupiñán, R. (2020). Nuestra herencia de maíz: Semillas nativas vs. semillas transgénicas, una aproximación desde la cultura y los derechos humanos' Revista Derecho y Realidad, 18, (35), 39-51. 


\section{NUESTRA HERENCIA DE MAÍZ: SEMILLAS NATIVAS VS. SEMILLAS TRANSGÉNICAS, UNA APROXIMACIÓN DESDE LA CULTURA Y LOS DERECHOS HUMANOS}

Our corn heritage: native and transgenic seeds, an approach from culture and human rights

Nossa herança de milho: sementes nativas vs. sementes transgênicas, uma abordagem da cultura e dos direitos humanos

\section{Yesica Ruiz-Cristancho}

Estudiante de Licenciatura en Artes Plásticas. Universidad Pedagógica y Tecnológica de Colombia - UPTC, Colombia

yesica.ruiz01@uptc.edu.co

\section{Ricardo Alonso Tejedor-Estupiñán}

Magister en Economía. Investigador Grupo Modeal Universidad Pedagógica y Tecnológica de Colombia- UPTC, Colombia. ORCID ID: orcid.org/0000-0002-5877-846X

ricardotse@hotmail.com

Recepción: 24 de noviembre 2019

Aceptación: 7 de junio 2020

\section{RESUMEN}

El propósito de este artículo de reflexión es resaltar la importancia que tiene el uso de las semillas nativas de maíz frente a las semillas transgénicas en el marco del derecho humano a tomar parte en la vida cultural de la comunidad, tanto para algunas poblaciones así como para algunos artistas. Se realiza un análisis crítico al respecto de cómo el uso de semillas nativas de maíz forman parte importante de las relaciones sociales y materiales en el marco de los derechos culturales de comunidades indígenas, campesinas, afro, etc., así como también para algunos artistas contemporáneos.

Se destacan algunos aportes que desde el arte y la cultura, permiten generar espacios para mantener vivas tradiciones, valores y prácticas que rondan el cultivo ancestral del maíz y sus múltiples usos.
Se concluye que la desaparición de las semillas nativas puede representar una pérdida irreparable de la riqueza material e inmaterial de dichas comunidades, por lo cual el arte y la cultura pueden convertirse en una herramienta de resistencia frente a las restricciones legales que algunos gobiernos promueven, al establecer políticas comerciales desacertadas de uso exclusivo de semillas transgénicas y que conllevan a la desaparición de las nativas.

\section{PALABRAS CLAVES}

Semillas nativas; semillas transgénicas; derechos humanos; Seguridad alimentaria; arte; cultura; performance.

\section{ABSTRACT}

The purpose of this reflection article is to highlight the importance of the use of 
native corn seeds compared to transgenic seeds in the framework of the human right to take part in the cultural life of the community, both for some populations as well as for some artists. A critical analysis is made regarding how the use of native corn seeds are an important part of social and material relations within the framework of the cultural rights of indigenous, peasant, afro communities, etc., as well as for some contemporary artists.

Some contributions that stand out from art and culture, allow generating spaces to keep alive traditions, values and practices, which haunt the ancestral cultivation of corn and its multiple uses. It is concluded that the disappearance of native seeds can represent an irreparable loss of the material and immaterial wealth of these communities, so that art and culture can become a tool of resistance against the legal restrictions that some governments promote, by establish misguided trade policies for the exclusive use of transgenic seeds and that lead to the disappearance of native ones.

\section{KEYWORDS}

Native seeds; transgenic seeds; human rights; food security; arts; culture.

\section{RESUMO}

O objetivo deste artigo de reflexão é destacar a importância do uso de sementes de milho nativas em comparação com sementes transgênicas no âmbito do direito humano de participar da vida cultural da comunidade, tanto para algumas populações quanto para alguns artistas. Uma análise crítica é feita sobre como o uso de sementes de milho nativas é uma parte importante das relações sociais e materiais no âmbito dos direitos culturais das comunidades indígenas, camponesas, afro, etc., bem como para alguns artistas contemporâneos.

Algumas contribuições que se destacam da arte e da cultura permitem gerar espaços para manter vivas tradições, valores e práticas que envolvem o cultivo ancestral do milho e seus múltiplos usos. Conclui-se que o desaparecimento de sementes nativas pode representar uma perda irreparável da riqueza material e imaterial dessas comunidades, para que a arte e a cultura se tornem uma ferramenta de resistência às restrições legais promovidas por alguns governos, estabelecer políticas comerciais equivocadas para o uso exclusivo de sementes transgênicas e que levem ao desaparecimento de sementes nativas.

\section{PALAVRAS-CHAVE:}

Sementes nativas; sementes transgênicas; direitos humanos; segurança alimentar; arte; cultura; performance.

\section{INTRODUCCIÓN}

Cada país está ubicado en un mosaico ecológico, el cual posee condiciones específicas geográficas, biológicas, climatológicas, etc., lo que conllevó a que las comunidades humanas se adaptaran a dichas condiciones haciendo de sus territorios la despensa de gran variedad de alimentos para las poblaciones que originalmente lo habitaron, desde antes de la aparición de los grandes consorcios comerciales relacionados con la industria agrícola a gran escala. En el caso del territorio sur americano, este fue el espacio del cual se sirvieron las poblaciones indígenas, rurales, campesinas y afro, producto del esclavismo colonial (Kohn, M., \& O’Neill, D. I. 2006).

Así mismo, las semillas nativas han sido la fuente principal de la que se han abastecido históricamente estas poblaciones para su sustento, y además forman parte inherente de los valores e identidad cultural, antes de la entrada e imposición estatal y comercial del uso de las semillas transgénicas.

El propósito del artículo, por tal razón, es presentar al lector la discusión de la desaparición silenciosa a la que se encuentran abocadas las semillas nativas, lo cual afecta no solo a las poblaciones campesinas, indígenas, afro, entre otras, sino también la soberanía alimentaria de la sociedad mayoritaria e incluso a algunos artistas quienes hacen uso de estas dentro 
de sus proyectos, integrando esta discusión dentro del derecho consignado en el artículo 27 de la Declaración Universal de Derechos Humanos que estipula:

1. Toda persona tiene derecho a tomar parte libremente en la vida cultural de la comunidad, a gozar de las artes y a participar en el progreso científico y en los beneficios que de él resulten.

2. Toda persona tiene derecho a la protección de los intereses morales y materiales que le correspondan por razón de las producciones científicas, literarias o artísticas de que sea autora. (General, L. A., 1948. p.4)

Teniendo en cuenta dicho escenario, donde algunos países están inmersos en acuerdos comerciales que promueven normas que privilegian el uso de semillas transgénicas y sus efectos negativos sobre las semillas nativas, se plantea el siguiente problema: ¿Salvaguardar el uso de semillas nativas y criollas, permite también garantizar el derecho humano a tomar parte en la vida cultural de la comunidad?

Ahora bien, se destaca entre todos los productos, la agricultura del maíz, debido a que es un cultivo ancestral que proviene de periodos inmemoriales y que además ha sido un producto elemental que ha satisfecho la necesidad de alimentarse, en especial a las actuales poblaciones americanas.

Además, a través de la tradición oral y de otras expresiones culturales de dichos pueblos, se ha transmitido la forma de cultivar los alimentos por generaciones, donde los valores como la cooperación, el respeto, el amor, el cuidado y el trabajo en la tierra con las semillas, son parte esencial para la supervivencia de las comunidades rurales que aún habitan en estos países.

Históricamente se ha destacado el uso de las semillas nativas o criollas como un producto del cual se han servido generaciones de comunidades agrícolas, fruto de domesticaciones que las han adaptado a sus ambientes, generando sistemas de producción sustentables para satisfacer sus necesidades. Estudios como los de Mickleburgh, H. y Pagán-Jiménez, J. demuestran, con base en investigaciones recientes, que los habitantes precolombinos de la región Caribe insular consumieron una dieta de amplio espectro donde el uso del maíz era más común de lo que se pensaba inicialmente y que además dicho consumo de maíz estuvo, en algunos casos, asociado con banquetes y actividades ceremoniales (2012 pág. 2468).

Por tal motivo, las semillas nativas han servido a las comunidades rurales campesinas, indígenas, afro, entre otras, para su sustento y también forman parte esencial de sus mitos, tradiciones $y$, en general, de su cultura. Por su parte, Ascencio Aguirre, N. y Melo López, D. (2017) muestran de forma detallada: como las prácticas de recuperación, uso, reproducción, intercambio y difusión del conocimiento de las semillas criollas y nativas, pueden aportar a la construcción de nuevas ciudadanías y de soberanía alimentaria en los territorios de Colombia.

Así mismo, es importante tener en cuenta el grado de vulnerabilidad económica en que se encuentran las poblaciones indígenas y campesinas, lo cual se ha convertido en un tema de interés para la salud pública en todo el mundo, al respecto se pueden tomar las ideas de Restrepo-Arango et al (2018) y Huet et al (2012).

Adicionalmente, es importante reconocer que las comunidades indígenas, campesinas, afro, etc., han sido, por generaciones, guardianas de la diversidad cultural y la riqueza natural de cada país; estos pueblos han conservado las semillas entre sus conocimientos y prácticas productivas ancestrales y agrícolas, donde el saber va de la mano con la semilla y esta depende no solo de su custodio sino de la comunidad, pues si esto no es así, sería una semilla vacía (Conocimiento Tradicional, 2017).

Aunque los derechos colectivos y prácticas culturales están hoy protegidos por convenios internacionales, la Constitución Política nacional y la ley, en muchos casos 
los derechos sobre sus territorios y recursos naturales son ignorados y vulnerados (Grupo Semillas, C. 2016).

Por otra parte, De Sousa Santos, B. (2009) quien ha buscado conceptos alternativos para generar diálogos entre culturas, considera que es posible generar nuevas epistemologías desde el sur como proyecto contra hegemónico de emancipación social para: "conducir a universalismos regionales o sectoriales construidos, partiendo desde abajo" (p. 358), dicho concepto lo denomina "cosmopolitismo subalterno o insurgente" y es una sugerencia del autor para resolver los conflictos y diálogos entre las diferentes formas de conocimiento que se dan, por ejemplo, en el caso de la agricultura industrial frente a la agricultura campesina (p.133).

Luego de esta breve presentación, el artículo continúa con dos secciones, la primera aborda una revisión de literatura que identifica algunos aspectos de discusión sobre el uso de semillas nativas frente a las transgénicas. La segunda sección da a conocer algunas expresiones y disertaciones de diversos artistas, los cuales promueven y defienden la autonomía del uso de las semillas nativas, en particular las de maíz, y de esta forma contribuir con la protección y garantía de este derecho humano universal que invita a la libertad de tomar parte en la vida cultural de la comunidad. Finalmente se presentan las respectivas conclusiones.

\section{SEMILLAS NATIVAS VERSUS SEMILLAS TRANSGÉNICAS}

No es difícil demostrar cómo distintas culturas realizaron, tiempo atrás, procesos de agricultura intensiva, los cuales permitieron la subsistencia de emergentes imperios que convocaron grandes poblaciones (Gulyás, S., \& Sümegi, P. 2011), empero la producción y el consumo de productos agrícolas en la actualidad, en el mundo, está sujeto principalmente al modelo de agricultura intensiva basada en el uso de pesticidas, agroquímicos y semillas transgénicas, amparado por convenios de política comercial acordados entre los países que forman parte de la Organización Mundial del Comercio y el Acuerdo sobre la Aplicación de Medidas Sanitarias y Fitosanitarias. Dicho acuerdo menciona a la Comisión Mixta FAO/OMS del Codex Alimentarius ${ }^{1}$ como la organización de normalización competente para los alimentos y a la CIPF $^{2}$ para la salud de las plantas. OMC (s.f)

Con respecto a los riesgos del uso para el consumo animal de dichos productos, según Blanco Martínez (2012), estas semillas pueden poseer en su estructura una 'contaminación por flujo de genes' que las hace vulnerables, por ende son cultivadas necesariamente con químicos. Por su parte, Cyran et al (2008), en un estudio de laboratorio realizado, revelan que los alimentos transgénicos son una amenaza para la fertilidad y también descubren que un maíz modificado genéticamente afecta a la reproducción en ratones.

Por su parte, Greenpeace España (2010), refiere que los riesgos sanitarios a largo plazo, de los organismos modificados genéticamente (OMG), presentes en nuestra alimentación o en la de los animales cuyos productos consumimos, no se están evaluando correctamente y que algunos informes científicos independientes muestran evidencias de riesgos a la salud tales como: aparición de nuevas alergias por introducción de nuevas proteínas en los alimentos.

En Estados Unidos, es muy bien el conocido caso del "Maíz Starlink"3, la

1. El Codex Alimentarius es una colección de normas internacionales en materia de inocuidad de los alimentos adoptadas por la Comisión del Codex Alimentarius (el "Codex"). El Codex tiene su sede en Roma y es financiado conjuntamente por la FAO y la OMS.

2. La Convención Internacional de Protección Fitosanitaria (CIPF) es un tratado multilateral para la cooperación internacional en la esfera de la protección fitosanitaria. La Convención elabora disposiciones para la aplicación de medidas por parte de los gobiernos con objeto de proteger sus recursos vegetales de plagas perjudiciales (medidas fitosanitarias) que pueden introducirse mediante el comercio internacional.

3. En dicho caso se encontraron en la cadena alimentaria, trazas de un maíz transgénico no autorizado para consumo humano que provocó graves problemas de reacciones alérgicas. 
aparición de resistencias a antibióticos en bacterias patógenas para el hombre, aparición de nuevos tóxicos en los alimentos, incremento de la contaminación en los alimentos por un mayor uso de productos químicos en la agricultura, entre otras evidencias claras de los riesgos en parámetros sanguíneos asociados con las funciones renales y hepáticas. Igualmente, se discute el uso de semillas como la variedad transgénica MON810 que se cultiva sobre todo en España, con el visto bueno de las autoridades europeas, quienes han permitido su importación para entrar en la alimentación humana y animal.

También se encuentran diferentes estudios científicos como los del neerlandés Henk Tennekes quien, junto con otros científicos, llevan más de dos décadas luchando contra consorcios como Bayer, demostrando como el uso de neonicotinoides -los cuales son pestiscidas adheridos a las semillas- son insecticidas peligrosos no solo para las plagas sino para otras especies como las abejas.

Para él y los demás científicos, dichos insecticidas deberían haber sido prohibidos hace tiempo, ya que en sus estudios advierten sobre los efectos negativos de los neonicotinoides. Además muestran como la industria, con la ayuda de autoridades gubernamentales, han detenido la prohibición de esas sustancias durante años ${ }^{4}$.

Así mismo, es conocido el reciente caso del jardinero estadounidense Dewayne Johnson, quien demandó a la empresa Bayer y su subsidiaria Monsanto, por considerarla responsable del cáncer terminal que padece a causa del uso del herbicida comúnmente conocido como glifosato Roundup y su versión profesional RangerPro, donde la empresa deberá indemnizarlo con la suma de 10.2 millones de dólares (D.W. 2020).

Por estas, entre otras razones, en Francia, Alemania y otros países europeos,

4. Al respecto se puede complementar la información con autores como Mason, R et al (2013) y Rondeau, G et al (2014) los agricultores y personas interesadas, apoyados por científicos y organizaciones ambientalistas, han adelantado algunas prohibiciones para el uso de ciertos pesticidas, semillas transgénicas y demás; igualmente se han gestado movimientos colectivos de resistencia de las semillas y tradiciones, como en el caso de Guatemala la comunidad Maya conocida como los Q'eqchi y se han discutido aspectos de la conciencia maya q'eqchi en relación con su praxis de sostenibilidad cultural, destacando las siguientes cinco dimensiones: 1 ). Conciencia rural indígena y resistencia histórica, 2). El modelo de economía basada en la subsistencia del sistema milpa, 3). Un paisaje de signos y símbolos de la cosmología maya, 4). El modelo ritual de la cosmogonía maya y los rituales agrícolas para la siembra y la curación y 5). Los campesinos indígenas se organizan para resistir y derrotar la Ley de Monsanto (Grandia, 2017).

Para el caso de México, que es un referente histórico y actual en la producción de maíz, Barrera-Bassols et al (2009) realizan un recuento de las acciones desarrolladas por la comunidad en defensa del maíz nativo frente a la llegada de maíz transgénico, en especial la defensa de su simbolismo, por ser el centro original de domesticación y diversificación de dicho grano, resaltan la necesidad de establecer una política de carácter nacional, en defensa de la soberanía alimentaria y la identidad cultural.

Actualmente, tomando el caso de Colombia, las semillas nativas o criollas se han visto sujetas a los efectos de la resolución 970, que fue remplazada por la resolución 3168 del ICA de 2015, la cual, fue una condición para la firma del Tratado de Libre Comercio con Estados Unidos en el año 2010 (Grupo Semillas, C. 2015a).

Por medio de esta resolución se ha criminalizado al campesino por considerar el uso de semillas nativas como una presunta piratería de semillas, lo cual es un hecho indignante, porque antes de la firma de este tratado comercial, las semillas transitaban libremente por las manos de agricultores, y tampoco era habitual el uso 
de agro-insumos o pesticidas, ya que existían formas naturales de control de plagas y un conocimiento vernáculo (Zerda, Á. 2003) que permitió la aplicación de una agricultura de supervivencia.

$\mathrm{Al}$ respecto se destaca la iniciativa de la Corporación Grupo Semillas Colombia, la cual busca fortalecer la defensa del uso de las mismas, haciendo conciencia de la seguridad alimentaria, ente otras dimensiones económicas, sociales, culturales y ambientales. Entre su actividad se encuentra haber hecho un análisis y discusión pública de leyes de propiedad intelectual que han logrado restringir el libre uso y circulación de las semillas, que hasta el momento habían sido producidas, conservadas, usadas, intercambiadas y comercializadas por nuestros antepasados.

La iniciativa de esta corporación tiene acciones de recuperación, conservación y defensa de semillas, como lo son los bancos de semillas vivos, trueques, rotación de cultivos con técnica de alelopatía, son las alternativas para apropiarse de esta invaluable herencia.

Por su parte, Trochez González et al. (2018), realizan un artículo en el cual muestran como Colombia, en su carrera por realizar acuerdos de libre comercio con diversos países y comunidades, liberó los aranceles de importación de varios productos agropecuarios, y en el caso del maíz, tales acuerdos generaron una disminución en la producción y una desmejora en los ingresos de los agricultores. Pero tales acuerdos, entre otras medidas tomadas por los últimos gobiernos, parecen no tener un efecto positivo sobre el crecimiento económico colombiano como lo demuestra Vallejo Zamudio (2017).

Por otro lado, un estudio realizado por Rodríguez Cervantes, A. (2014), deja expuesto cómo ha ido aumentando el control corporativo de las semillas generando competencia desigual concentrada en diez empresas multinacionales.
Esta situación generada por derechos de propiedad intelectual, las cuales privilegian los intereses de las multinacionales que ya acaparan el mercado internacional de semillas como Monsanto, Dupont, Bayer, Syngenta, entre otras, considera a toda semilla que no sea certificada por el ICA como una semilla ilegal. Estas reglas de juego ponen en riesgo no solo la seguridad alimentaria de las poblaciones sino también el valor cultural de esta herencia de la naturaleza.

Para finalizar esta sección, se puede evidenciar que según los autores mencionados, existen diferentes consecuencias negativas derivadas de este tipo de agricultura, tanto económicas como sobre la salud y la integridad cultural. En torno a las económicas, no solo incrementando la precariedad laboral en las comunidades rurales del país sino fomentando el desplazamiento de la producción nacional por la importación de productos agrícolas. Sobre la salud, lo cual está demostrado en diferentes estudios que corroboran los efectos dañinos del uso de productos asociados a la agricultura transgénica a gran escala, e igualmente sobre los derechos culturales, en especial aquellos referidos en este escrito.

Lo anterior se debe a que la legislación nacional e internacional coacciona a los agricultores para utilizar semillas certificadas, buscando estandarizar el mercado (Chiquin, 2014), sin tener en cuenta el valor simbólico que tienen las semillas nativas para los campesinos, ni la importancia que poseen tales semillas para la cultura de una población.

\section{ALGUNOS APORTES DESDE EL ARTE Y LA CULTURA}

Como el objetivo de este artículo es también incluir la visión que se ofrece desde el arte a la problemática expuesta, en este caso, el uso de las semillas nativas frente a las semillas transgénicas, que comercializan los grandes oligopolios, se traen a colación algunas expresiones culturales y representaciones de artistas que buscan 
rechazar tales prácticas comerciales que homogenizan y conllevan a desaparición de variedades de semillas en especial, la semilla de maíz.

Por su parte, Damián, M., y Alberto, M. (2010) explican las diversas ideas mayas de la creación del hombre en un estudio basado en textos mayas coloniales - específicamente el Popol Vuh, los libros de Chilam Balam y el Ritual de los Bacabes-. Aquí los autores retoman un fragmento del Popol Vuh donde "se narra la creación del hombre a partir del maíz blanco y amarillo. El maíz viene a ser la síntesis de la abundancia y el hombre, por tanto, el producto de la riqueza de los recursos de su medio ambiente" (p. 281).

También resaltan que para los mayas existió la creencia que "la población humana coexiste con la población vegetal, animal, mineral y meteórica en un intercambio biológico que se expresa en mitos y rituales específicos. Para el pensamiento maya, cuerpo y naturaleza son un solo territorio, el territorio de lo sagrado" (p. 279). Esta concepción, sacralizada entre el hombre y su relación con lo no humano -reino vegetal, animal, fungi- puede ser desestimada por el pensamiento y la visión de progreso que determina el hombre moderno.

Al respecto, Sabino Aguilar (2019) desarrolla un completo análisis desde un abordaje conceptual antropológico de las antiguas sociedades agrarias en algunas civilizaciones antiguas, su investigación explica, de forma detallada, basado en anteriores investigaciones de reconocidos antropólogos e historiadores, los mitos asociados al maíz desde la cosmovisión andina y las culturas precolombinas, de Perú, Bolivia y Chile.

También se encontró el trabajo de María Teresa Hincapié, un artista reconocida principalmente por ser una de las pioneras del performance en Colombia (Roca, 2008). En este sentido, Hincapíe (2006), en una entrevista realizada para la revista de Psicoanálisis - Desde el Jardín de Freud, deja entrever su idea de la relación entre el arte y la naturaleza:
Creo que una obra de arte es un regalo y no una mercancía. El arte, según los principios, ha estado ligado a la vida. $\mathrm{Y}$ la vida no es efímera, es el tiempo, es el tiempo de la naturaleza, y hay muchas maneras de expresarlo: el pintor con su pintura, el bailarín... Es la época en que estamos viviendo que exige que eso sea así, de volver a amar las cosas esenciales, las cosas importantes frente al consumismo [...]. La industrialización, la comercialización y el consumo... nos han llevado a una pérdida de valores [y] del sentido de la vida.

Por otro lado, se encuentra a Fernando Pertuz, un artista del performance colombiano, quien considera que el cuerpo es la excusa para entablar discusiones, diálogos, reflexiones con la sociedad, y así sentir estos acercamientos plásticos. Desde esta perspectiva, el dialogo con el cuerpo, es la enfermedad la que muestra los deterioros causados entre otros, por nuevos hábitos alimenticios.

Así mismo, otra artista que ha trabajado con custodios boyacenses es María Buenaventura. Ella, a diferencia de los artistas anteriormente mencionados, trabaja la instalación como medio de persuasión artística a esta problemática. Una de sus obras más conocidas es el observatorio de maíz hecha de la mano con el custodio Fabriciano Ortiz; Buenaventura realiza una reflexión interesante al respecto:

Alejada momentáneamente de las angustias por las amenazas a la existencia de estas variedades de maíz nativas de la región cundiboyacense y del campesinado colombiano, causados por las políticas de monocultivo $\mathrm{y}$ la industria alimentaria, el ejercicio invita a disfrutar la diversidad de formas y colores, la enorme belleza de la "imperfección", es decir, de aquellas semillas que no son homogéneas y regulares, como se requiere en los procesos industriales (SIC). En resumen la variabilidad que permite crear la vida en la tierra...La observación lleva en 
2017 a una relación entre tres creaciones de las culturas originarias: los tunjos de oro, las vasijas de barro y los granos de maíz. Tres formas surgidas de un mismo pensamiento. (Buenaventura, M. 2017, s.p.)

William Stark (2020) por su parte, introduce su investigación a través de la investigación analítica reflexiva, en una variedad de consideraciones centrales referente a discusiones de la importancia del maíz sobre la historia, la sociedad y la cultura mesoamericana y estadounidense, a través del arte de performance de la mexicana Violeta Luna, quien también en otros trabajos como NK603: Action for Performer \& e-Maíz (NK603) realiza una reflexión crítica sobre las consecuencias devastadoras del consumo de maíz genéticamente modificado sobre la vida.

Por otra parte, se encuentra el maestro autodidacta, Ismael Manco quien es un artista campesino de Duitama, una pequeña ciudad del departamento de Boyacá (Colombia). Sus esculturas, pinturas, dibujos e instalaciones las realiza con materiales naturales como tierras y tintes que se extraen de algunas semillas. Su proyecto artístico incluye generar "espacios de discusión y pensamiento frente a temas como la memoria de su territorio, su patrimonio natural y cultural, sus semillas nativas, y la herencia indígena de campesinas/os cundiboyacenses (habitantes de Cundinamarca y Boyacá)" (University of Texas Press, 2016).

Él mismo explica su proyecto así:

Soy agricultor, vivo de la agricultura en un terreno de mi familia que ha permanecido con nosotros por mucho tiempo, quizá unas 5 generaciones. Allí cultivamos semillas nativas con prácticas ancestrales. A lo largo de la historia, nuestro territorio ha sido de vocación agrícola, de hecho es la despensa de Colombia, puesto que un gran porcentaje de los alimentos que le quitan el hambre a este país provienen de nuestro territorio. Nosotros somos campesinos, agricultores y poseedores de una de las regiones en términos ambientales más importantes del mundo. Cerca del $50 \%$ de los páramos del planeta está en Colombia. Boyacá es el departamento del país con mayor área de páramo, y las políticas extractivistas están poniendo en riesgo estos ecosistemas y, de paso, las culturas ancestrales campesindias (SIC). Por eso comparto e intercambio semillas con personas (campesinos y no campesinos) comprometidas con procesos ambientales y con la defensa de las semillas nativas. Una semilla puede ser una palabra o un pensamiento; puede ser muchas cosas, y bueno, lo importante es sembrarla. (University of Texas Press, 2016).

Para finalizar, se destaca cómo las semillas de maíz son un símbolo de identidad cultural, no solo para comunidades campesinas, indígenas, afro sino que también son un elemento para la producción cultural de algunos artistas contemporáneos y modernos. Son una herencia ambigua que ha sobrevivido hasta la actualidad, gracias no solo a los métodos de conservación de estas, sino también a la tradición oral, así como la realización de performances, obras pictóricas y demás formas de arte abstracto contemporáneo.

Los artistas contemporáneos pueden aportar a las comunidades rurales campesinas indígenas, afro y demás actores en riesgo (Greenpeace México, 2013), por lo tanto, también son actores clave que han venido apoyando procesos de resistencia frente a las multinacionales quienes, en su afán de lucro, no consideran los efectos adversos de los productos industriales agrícolas y sus prácticas comerciales sobre la cultura de los pueblos.

Es preciso fortalecer, conservar $\mathrm{y}$ defender tradiciones así como el patrimonio natural, tal como lo plantea Rodríguez Cervantes, A. (2013), para poder definir y dar forma al espíritu de una época que recupere la identidad de una nación diversa, rica culturalmente, donde se puede decir orgullosamente que se posee una autonomía para conservar no solo valores y tradiciones 
sino que se pueda llegar a conservar también la diversidad de especies animales y vegetales que la naturaleza le ha entregado a cada territorio.

La actividad agrícola como proceso esencial para la supervivencia humana, ha encontrado en los horizontes del arte un soporte para expresar que: es la vida lo que está en riesgo! no solo la dela actual población, sino que también de las generaciones futuras, en caso tal de que los productores y oligopolios de semillas sean quienes determinen qué, quienes y en dónde producir.

\section{CONCLUSIONES}

Las diversas comunidades rurales habían hecho uso libre de sus propias semillas hasta que algunos gobiernos impusieron las barreras comerciales legales, en algunos casos, dichas comunidades están organizadas y reclaman su derecho a su libre uso. Por otra parte, entre los actores que defienden el uso de semillas nativas también se encuentran algunos artistas, quienes también aportan de forma importante desde sus representaciones discursivas y artísticas por cambiar esta dinámica.

Hasta el momento, gran parte de la población del planeta sigue ignorando esta problemática, creyendo que la ciencia a través de la genética, ha dado una solución completa al problema de la producción de alimentos y control de plagas, no obstante se están generando problemas, económicos y de salud, como ya se vio y no solo eso, sino que se está relegando el conocimiento y riqueza, asociado al uso de la semillas criollas y nativas que son parte de la identidad e historia de cada comunidad, afectando los derechos culturales establecidos en la carta de los derechos humanos.

Se requiere entonces que los estados reconozcan y garanticen el uso de semillas nativas a los pueblos indígenas, afro, campesinos y, en general, al resto de su población. Se requiere también propiciar y fortalecer espacios culturales donde la sociedad pueda reconocer su riqueza material e inmaterial, vegetal y animal. Los procesos llevados en estos escenarios muestran cuán importante es el arte contemporáneo para el reconocimiento de las semillas nativas como pensamiento y vida natural y la reconstrucción y fortalecimiento de una identidad basada en el respeto por la vida, la dignidad y la efectividad de los derechos.

La desaparición de las semillas nativas puede representar una pérdida importante de la riqueza material e inmaterial que detentan algunas comunidades, por lo cual, el arte y la cultura pueden convertirse en una herramienta de resistencia frente a las presiones legales que algunos gobiernos promueven al defender políticas comerciales en favor de las empresas multinacionales agrícolas. Ya para finalizar, debe considerarse que sembrar semillas nativas permite garantizar el derecho humano a tomar parte en la vida cultural de la comunidad, es sembrar soberanía y diversidad.

AGRADECIMIENTOS: Este artículo se desarrolló sin ningún financiamiento, forma parte del proyecto artístico y profesional de la autora. Los autores agradecemos los comentarios de los evaluadores anónimos de la revista. Finalmente los autores no declaran ningún conflicto de interés. 


\section{REFERENCIAS}

» Aguilar, S. A. (2019). Simbología del maíz en la cultura andina milenaria: resistencia e identidad del hombre andino. Investigaciones sociales, 22(41), 37-55. Disponible en: https://doi.org/10.15381/is.v22i41.16756.

» Ascencio Aguirre, N. P., \& Melo López, D. P. (2017). Soberanía alimentaria y nuevas ciudadanías a partir de las y los custodios de semillas (Doctoral dissertation, Corporación Universitaria Minuto de Dios).

» Barrera-Bassols, N., Astier, M., Orozco, Q., \& Schmidt, E. B. (2009). Saberes locales y defensa de la agrobiodiversidad: maíces nativos vs. maíces transgénicos en México. En: Papeles, 107, 77-91.

» Blanco Martínez, J. T. (2012). Monitoreo del flujo de genes de cultivos transgénicos de maíz a razas locales y variedades comerciales de maíz en el Valle de San Juan, Tolima (Doctoral dissertation, Universidad Nacional de Colombia).

» Buenaventura, M. (2017). Observatorio de maíz. [online] Disponible en: https://mariabuenaventura.com/portfolio/observatorio-de-maiz/ [Accessed 24 Mar. 2017].

» Cyran, N., Gülly, C., Handl, S., Hofstätter, G., Meyer, F., Skalicky, M., \& Steinborn, R. (2008). Biological effects of transgenic maize NK603xMON810 fed in long term reproduction studies in mice. Unpublished report: Institute fur Ernahrung, Austria.

» Chiquin, C. (2014). NO a la Ley Monsanto: Semillas para los pueblos, no para las empresas. Prensa Comunitaria, Km, 169.

» Conocimiento Tradicional (2017). Custodios de Semillas / Colombia. [video] Disponible en: https://www.youtube.com/watch?v=9EV9cfPvEnw.

» Damián, M., \& Alberto, M. (2010). Territorio sagrado: cuerpo humano y naturaleza en el pensamiento maya. En: Cuicuilco, 17(48), 279-298.

» De Sousa Santos, B. (2009). Una epistemología del sur: la reinvención del conocimiento y la emancipación social. Siglo XXI.

» ED.W. (2020). Bayer pierde apelación en caso de cáncer por Roundup. Disponible en: https://www.dw.com/es/bayer-pierde-apelaci\%C3\%B3n-en-caso-dec\%C3\%A1ncer-por-roundup/a-54250603.

» Huet, C., Rosol, R., \& Egeland, G. M. (2012). The prevalence of food insecurity is high and the diet quality poor in Inuit communities. En: The Journal of nutrition, 142(3), 541-547. DOI: 10.3945/jn.111.149278.

» General, L. A. (1948). Declaración Universal de los Derechos humanos. Naciones Unidas.

» Grandia, L. (2017). Sacred Maize against a Legal Maze: The Diversity of Resistance to Guatemala's' Monsanto Law'. En: Journal for the Study of Religion, Nature \& Culture, 11(1).

» Greenpeace España. (2010). Efectos para la salud. [online] Disponible en: http://archivo-es.greenpeace.org/espana/es/Trabajamos-en/Transgenicos/ Transgenicos/Problemas-de-los-transgenicos/Efectos-de-los-transgenicos-parala-salud1/.

Greenpeace México. (2013). Artistas por el maíz. ¡No al maíz transgénico! [vi- 
deo] Disponible en: https://www.youtube.com/watch?v=H0i8HWx4vVg

» Grupo Semillas, C. (2013). Las leyes que privatizan y controlan el uso de las semillas, criminalizan las semillas criollas. En: Revista Biodiversidad, 78. Disponible en: http://www.semillas.org.co/es/las-leyes-que-privatizan-controlan-el-uso-delas-semillas-y-criminalizan-las-semillas-criollas.

» Grupo Semillas, C. (2016). Campaña Semillas de Identidad. En defensa de la biodiversidad y la soberanía alimentaria - Semillas. [online] Semillas.org.co. Disponible en: http://www.semillas.org.co/es/campanas/campa-3 [Accessed 24 Oct. 2017].

» Grupo Semillas, C. (2015). Las leyes de semillas aniquilan la soberanía y autonomía alimentaria de los pueblos. Bogotá: Grupo Semillas. En: Retrieved on, 4(02).

» Grupo Semillas, C. (2015a). La resolución 3168 Del ICA de 2015 sobre semillas reemplaza la resolución 970. En: Revista Semillas, 61(62), 1-6.

» Gulyás, S., \& Sümegi, P. (2011). Farming and/or foraging? New environmental data to the life and economic transformation of Late Neolithic tell communities (Tisza Culture) in SE Hungary. En: Journal of Archaeological Science, 38(12), 33233339.

» Kohn, M., \& O'Neill, D. I. (2006). A tale of two Indias: Burke and Mill on empire and slavery in the West Indies and America. En: Political Theory, 34(2), 192-228.

» Luna, Violeta. 2015. NK603: Action for performance \& e-Maiz. Unpublished presentation at California State University Los Angeles. Disponible en https://dialnet.unirioja.es/servlet/articulo?codigo $=7461140$.

» Mason, R., Tennekes, H., Sánchez-Bayo, F., \& Jepsen, P. U. (2013). Immune suppression by neonicotinoid insecticides at the root of global wildlife declines. En: $J$ Environ Immunol Toxicol, 1(1), 3-12.

» Mickleburgh, H. L., \& Pagán-Jiménez, J. R. (2012). New insights into the consumption of maize and other food plants in the pre-Columbian Caribbean from starch grains trapped in human dental calculus. En: Journal of Archaeological Science, 39(7), 2468-2478.

» OMC (s.f). La OMC y la Organización para la Agricultura y la Alimentación (FAO). Disponible en: https://www.wto.org/spanish/thewto_s/coher_s/wto_fao_s.htm.

» Restrepo-Arango, M., Gutiérrez-Builes, L. A., \& Ríos-Osorio, L. A. (2018). [RETRACTED ARTICLE]: Food security in indigenous and peasant populations: a systematic review. En: Ciencia \& saude coletiva, 23(4), 1169-1181.

» Roca, J. (2008). Maria Teresa Hincapié (1954 - 2008) - [esferapública]. [online] Esferapublica.org. Disponible en: http://esferapublica.org/nfblog/maria-teresahincapie/ [Accessed 24 Oct. 2017].

» Rodríguez Cervantes, A. (2013). El despojo de la riqueza biológica: de patrimonio de la humanidad a recurso bajo soberanía del Estado. Heredia, Costa Rica: Universidad Nacional. EUNA.

» Rodríguez Cervantes, A. (2014). El control corporativo de las semillas y sus consecuencias más allá de la agricultura. En: C. Toro Pérez, E. Bravo, \& G. Vélez, La ecología política de la bioseguridad en América Latina (págs. 59 -81). Bogotá, Colombia: Universidad Nacional de Colombia, sede Bogotá. 
» Rondeau, G., Sánchez-Bayo, F., Tennekes, H. A., Decourtye, A., Ramírez-Romero, R., \& Desneux, N. (2014). Delayed and time-cumulative toxicity of imidacloprid in bees, ants and termites. En: Scientific reports, 4, 5566. Disponible en: https://doi. org/10.1038/srep05566.

» Stark, W. R. (2020). Violeta Luna: Representaciones de la identidad indígena: la mujer del maíz-la llorona cibernética. En: Contextos: Estudios de humanidades y ciencias sociales, (45), 8.

» University of Texas Press (2016). El arte de José Ismael Manco Parra. En: Diálogo 19(1), 184-224. Disponible en: 10.1353/dlg.2016.0048.

» Trochez González, J., Valencia Cárdenas, M., \& Salazar Uribe, J. (2018). Los efectos del Tratado de Libre Comercio con Estados Unidos y los precios del maíz colombiano. En: Apuntes Del Cenes, 37(65), 151-172. Disponible en: https://doi. org/10.19053/01203053.v37.n65.2018.5988.

»Vallejo-Zamudio, L. (2017). El incierto crecimiento económico colombiano. En: Apuntes Del Cenes, 36(64), 9-10. Disponible en: https://doi. org/10.19053/01203053.v36.n64.2017.6511.

» Zerda, Á. (2003). Propiedad intelectual sobre el conocimiento vernáculo. Bogotá, Universidad Nacional de Colombia. 